\title{
How does pre-reduction MRI affect surgeons' behaviour when reducing distraction-flexion (dislocation) injuries of the cervical spine?
}

\author{
Dr MA Fleming MBBCh, FCOrth(SA), MMed \\ Orthopaedic Surgeon, Groote Schuur Hospital, University of Cape Town \\ Dr T Westgarth-Taylor MBChB, FC Rad Diag(SA) \\ Senior Radiology Registrar, Groote Schuur Hospital, University of Cape Town \\ Dr S Candy BSc, HED, MBChB(UCT), FCRad(D)(SA) \\ Head of Radiology Department, Groote Schuur Hospital, University of Cape Town \\ Professor R Dunn MBChB(UCT), FCS(SA)Orth, MMed(UCT)Ortho \\ Consultant Spine and Orthopaedic Surgeon, Professor and Head of Department of Orthopaedic Surgery, \\ University of Cape Town, Groote Schuur and Red Cross Children's Hospitals \\ Corresponding author: \\ Professor R Dunn \\ Groote Schuur Orthopaedic Department \\ Telephone: $+27(0) 214045118$ \\ Fax: $+27(0) 214472709$ \\ Email: robert.dunn@uct.ac.za
}

\begin{abstract}
Study design: Retrospective review

Aim: To identify factors affecting surgeons' management decisions regarding acute cervical distraction-flexion dislocation reduction and the consequences thereof.

Summary of background data: There is clinical benefit when early ( $<24$ hours) decompression in distraction-flexion dislocation (DF) injuries with cord injury is performed. The risk of secondary cord injury during awake closed reduction is low. The need for MRI scanning prior to reduction is controversial but it may identify patients with an uncontained herniated 'disc at risk' that may be drawn into the spinal canal during reduction, causing further cord injury. Surgeons' belief regarding the importance of pre-reduction MRI varies. Thus in many clinical scenarios, treatment algorithms are chosen individually by the surgeon on the merits of each case as well as limited access to MRI facilities in the remoter areas of this large country.
\end{abstract}

Methods: Analysis was performed on 110 consecutive patients with a mean age of 37.1 years with DF dislocation injuries of the cervical spine. Pre-reduction MRI scans were assessed by two independent, blinded teams to determine patients with a 'disc at risk'. This subgroup was then investigated as to the management decisions, neurological status and outcome.

Results: Nineteen patients $(21 \%)$ were identified to have a perceived 'disc at risk'. Six of these patients underwent anterior surgery. Initial closed reduction was attempted in the other 13. None deteriorated neurologically. Presenting neurological status was found to have a large impact on surgeons' choice of reduction. Of the nine ASIA A patients, seven had initial closed reduction, while in the three ASIA E group only one had closed reduction.

Conclusion: Patients with agreed MRI features of a perceived 'disc at risk' had no increased risk of secondary cord injury. The presence of these disc lesions only influenced our surgeons to choose open reduction in four cases (21\%). Neurological status had a much greater effect on surgical decision-making in that those with neurological deficit (most to gain) were reduced closed and ASIA E (most to lose) tended to open reduction. Early reduction need not wait for MRI imaging and should be performed as soon as possible in cord-injured patients.

Key words: cervical, MRI, distraction-flexion, unifacet, bifacet, surgeon behaviour 


\section{Introduction}

Cervical spine dislocation carries an inherent risk of cord injury. Although we have little influence over the primary injury, we can improve outcome by limiting the secondary effects of ongoing compression and physiological circumstances which lead to ischemia and cellular changes of the cord. ${ }^{1,2}$ Animal models confirm that persistent cord compression has time-related adverse effects on the ability to recover. ${ }^{2,3}$ Clinical benefit has been demonstrated with cervical reduction performed earlier than 24 hours following injury. ${ }^{4}$

The safety of closed reduction has been questioned with sporadic case reports of secondary cord injury occurring with unrecognised cervical disc prolapse. ${ }^{5-9}$ Eismont and Green concluded that pre-reduction MRI will identify cervical disc prolapse thus allowing the surgeon to avoid secondary cord injury during reduction. Evidence of a prolapsed disc might change clinical management by dictating an immediate anterior decompression.

Although the incidence of disco-ligamentous lesions in distractive flexion injuries has been reported to be as high as $18 \%-80 \%,{ }^{10-12}$ the incidence of secondary cord injury following closed reduction is considered low. ${ }^{13}$

Thus a discrepancy exists between identifiable disc lesions on MRI and clinical significance. Reporting of disc lesions does not carry a high interrater reliability. ${ }^{12} \mathrm{MRI}$ is often not readily available due to distances involved between district hospitals and academic centres with MRI facilities and competition for resources. These factors may result in delay to cervical reduction with possible reduced neurological outcome.

Treatment pathways chosen by surgeons have been demonstrated to be inconsistent. ${ }^{14}$ Some surgeons perform pre-reduction MRI scans on all DF dislocation injuries, whereas others perform closed reduction on selected patients based on neurological status and stage of DF injury.

For this reason we performed this study to identify our surgeons' behaviour as regards the reduction decisionmaking process and clinical consequences thereof.

\section{Aim}

This retrospective review evaluates the management behaviour of our surgeons when faced with decisionmaking in acute DF dislocation injuries of the cervical spine, particularly with reference to the impact of MRI features, and the presenting neurological status of the patients.

\section{Methods}

Following local Institutional ethics approval (HRC Ref: 091/2011), 110 consecutive DF injuries were identified from the senior author's database. All were managed at a single tertiary hospital over a ten-year period.
An initial study ${ }^{12}$ was performed to assess the interrater agreement of MRI variables between radiologists and orthopaedic surgeons. The highest agreement was found on the presence of posterior disc herniation (based on defined posterior, inferior and corner-to-corner lines) and containment variables. These variables were then used to define the perceived 'disc at risk' - a herniated uncontained disc that may cause secondary cord injury during cervical reduction techniques. Both teams were blinded to patients' clinical data and worked completely independently.

Nineteen of the 110 MRI DF dislocation injuries satisfied both teams that there was the presence of a 'disc at risk'. Clinical data of this subgroup was then assessed. Case notes were reviewed, noting the management algorithm chosen and reasons for doing so. When employed, closed reduction involved skeletal traction applied with skull tongs to the awake patient. Sequential weights were added while visualising the cervical spine in a slightly flexed position with an image intensifier. The patient was monitored clinically for onset of neuralgia or weakness. Once the facets had been distracted sufficiently the head was gently repositioned in extension; the weights were reduced when the facets were visualised to be reduced. Following reduction, the patient was kept in the extended position in skeletal traction until definitive surgical fixation was performed. Should open reduction be performed, an anterior Smith-Robinson cervical approach was utilised and a discectomy performed prior to reduction. ${ }^{15,16}$ Frequently skull traction was combined with a levering manoeuvre using a MacDonald or Cobb instrument in the inter-body space on the side of the dislocation (Cloward/ reverse-Cloward manoeuvre). A cage or structural allograft was placed in the disc space and anterior locked plating performed..$^{17,18}$

Chronic injuries are not applicable to closed reduction. Patients who presented with a delay of more than three weeks from time of injury $(n=15)$ and patients whose clinical notes were missing $(n=6)$ were excluded from data analysis.

Data was collected and entered utilising a double-entry method thereby reducing entry error. ${ }^{19}$ Epidata and STATA statistical software was used.

\section{Results}

\section{'Disc at risk' cases}

Nineteen patients (21\%) had a perceived 'disc at risk' based on uncontained, posteriorly herniated intervertebral disc based on independent, blinded assessment by both radiologists and orthopaedic surgeons (Figure 1).

Of these 19 patients, six had anterior discectomy and reduction performed primarily. Four of these were operated upon due to the surgeon recognising MRI features of a disc prolapse. One was done due to the surgeon finding the patient to be neurologically intact and thus being concerned over the possibility of neurological deterioration, and one patient for an undocumented reason. 


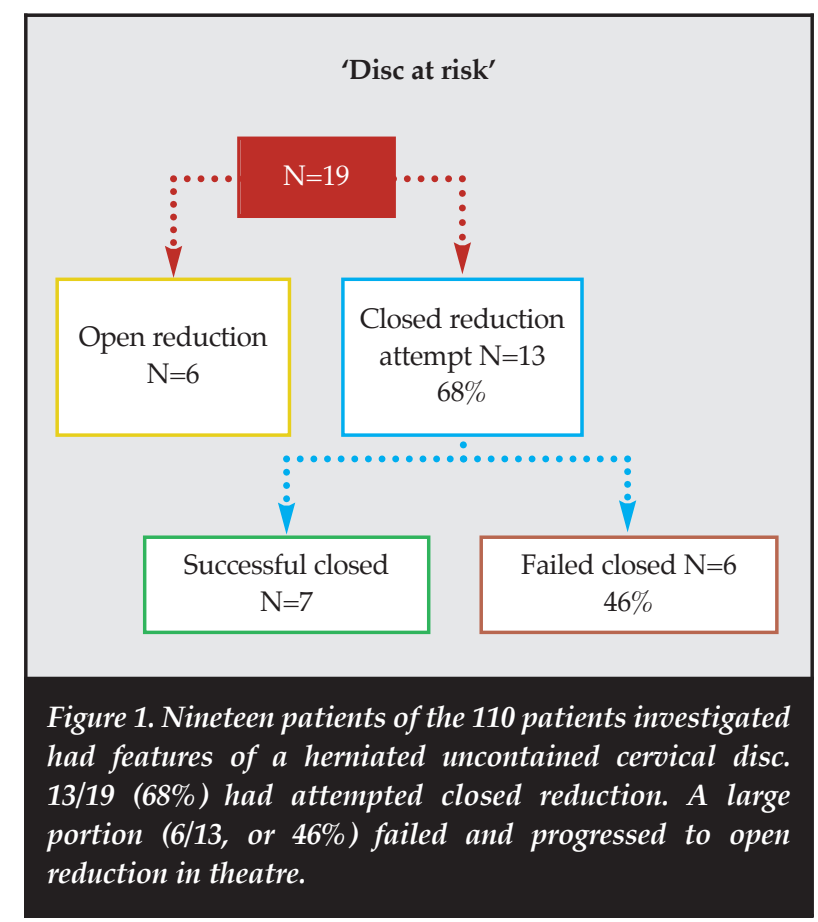

The remaining 13 patients had an attempted closed reduction despite the presence of the 'disc at risk' on MRI. Six $(46 \%)$ of these failed to reduce, at which point attempts were aborted in favour of open reduction. There was no secondary cord injury in any of these patients.

When assessing neurological status against the management chosen, there was a strong correlation between initial neurological impairment and the likelihood of the surgeon initially choosing closed reduction (Figure 2).

Data was collected and entered utilising a double-entry method thereby reducing entry error
Of the nine patients who were neurologically complete (ASIA A), seven had attempted closed reduction. Of the seven who were neurologically incomplete (ASIA B/C/D), five had attempted closed reduction. Of the three patients who were neurologically intact, two had initial open discectomy and reduction.

\section{Neurological deterioration}

Of the 89 patients included in the total data analysis (early presenting cases/with notes available), 54 had an initial attempted closed reduction. One patient deteriorated following aborted closed reduction (1.8\%); this patient was assessed as motor-sensory complete (ASIA A) prior to attempted reduction. During the procedure the patient lost two MRC grades in the C 8 myotome $(5 / 5$ to $3 / 5)$. This patient had a unifacet dislocation and did not have agreed features of a 'disc at risk'.

\section{Discussion}

With evolving evidence that the risk of secondary cord injury during awake reduction for distraction flexion injuries is low, there has been a resurgence of intent to reduce these injuries closed acutely., ${ }^{7,1,11,20-22}$

Fehlings et al. reported significant neurological improvement six months after spinal cord injury, if adequately decompressed within 24 hrs of injury. ${ }^{4}$ These authors consider closed reduction, demonstrated with MRI scanning, as adequate decompression.

MRI scanning consumes time that could be spent decompressing the cervical spine. The interpretation of the MRI may at times be a source of disagreement and may steer orthopaedic surgeons towards operative reduction, if relying on radiologists' reporting exclusively ${ }^{12}$ which may introduce delays to conclusive reduction. MRI may demonstrate disc lesions well; however, the relevance of these disc lesions with regard to the risk of secondary cord injury is thrown into question by our findings and others. ${ }^{23}$

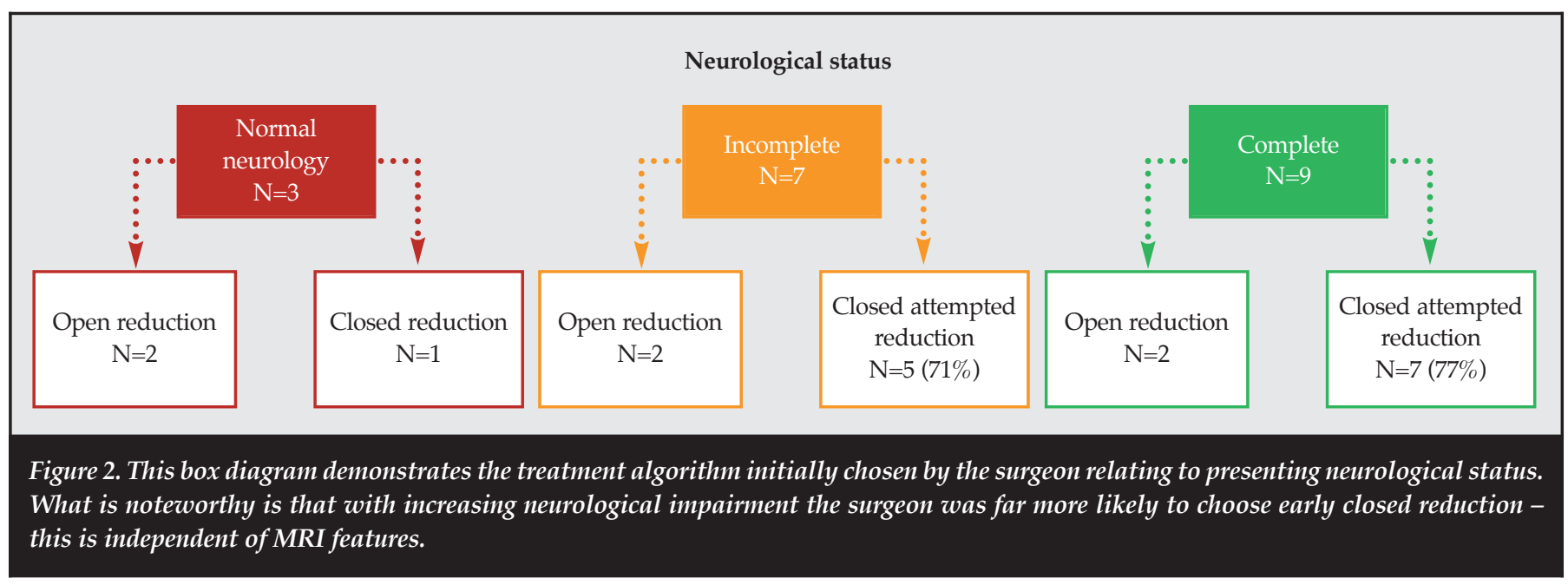


Despite agreed features of a 'disc at risk', 13 patients underwent attempted closed reduction and none deteriorated neurologically.

What we may conclude from our findings is that these 'disc at risk' injuries predict a high likelihood of failed closed reduction $(46 \%)$, as opposed to $2.6 \%$ failed reduction rate in other studies. ${ }^{13}$

We also note the high incidence of presenting neurological impairment in this subgroup. Patients with DF dislocation injuries and uncontained herniated discs had an $89 \%$ incidence of neurological compromise on presentation. This is in keeping with the understanding that the original injury was more severe with greater initial displacement when disc herniations are present. This finding is in keeping with recent level 3 evidence linking poorer neurological outcomes with an increasing stage of DF injuries. ${ }^{24}$

In this study we find that our surgeons' choice to attempt closed reduction initially was influenced greatly by the presence of initial neurological deficit and far less by the presence of a 'disc at risk' lesion. The principle of continued compression in an injured swelling cord steers our surgeons to choose early closed reduction. This may be based on the neurologically impaired being assessed as those with the 'most to gain' with early reduction (indirect decompression) and the neurologically normal, the 'most to lose'. Closed reduction is performed in the emergency room as described above.

The principle of continued compression in an injured swelling cord steers our surgeons to choose early closed reduction

The need to perform pre-reduction MRI is questioned, especially when it risks causing a delay to reduction in areas where MRI is relatively inaccessible. MRI may be best performed following reduction to confirm decompression. ${ }^{4}$

This remains a difficult question to answer in wellresourced environments where MRI is immediately available and the costs of routine pre-reduction MRI is compared to the rare post-reduction neurological deterioration.

\section{Acknowledgments}

Gen Chang - clerical work, Department of Radiology, Groote Schuur Hospital

Henri Carrara - statistical advice, University of Cape Town

No disclosures are pertinent to this study. Institutional ethics approval was obtained from the UCT Ethics Committee.

\section{References}

1. Tator $\mathrm{CH}$, Fehlings MG. Review of the secondary injury theory of acute spinal cord trauma with emphasis on vascular mechanisms. J. Neurosurg 1991;75:15-26.

2. Carlson GD, Gorden CD, Oliff HS, et al. Sustained Spinal Cord Compression Part I: Time-Dependent Effect on Long-Term Pathophysiology. J Bone Joint Surg Am. 2003;85:86-94.

3. Tarlov IM. Spinal cord compression studies III. Time limits for recovery after gradual compression in dogs. Neurol Psychiatry 1954;71:588-97.

4. Fehlings MG, Vaccaro A, Wilson JR, et al. Early versus delayed decompression for traumatic cervical spinal cord injury: results of the Surgical Timing in Acute Spinal Cord Injury Study (STASCIS). PLoS One 2012;7(2):e32037. doi:10.1371/journal.pone.0032037

5. Eismont FJ, Arena MJ, Green BA, et al. Extrusion of an intervertebral disc associated with traumatic subluxation or dislocation of cervical facets . Case report Traumatic of an Intervertebral Subluxation Disc Associated of Cervical with Facets or Dislocation of five. J Bone Joint Surg Am 1991;73:1555-60.

6. Berrington N, Staden J., Willers J, van der Westerhuizen J. Cervical intervertebral disc prolapse associated with traumatic facet dislocations. Surg Neurol 1993;40:395-99.

7. Lee AS, Maclean JCB, Newton DA. Rapid traction for reduction of cervical spine dislocations. J Bone Joint Surg Br. 1994;76(3):352-56.

8. Mahale Y, Silver J, Henderson N. Neurological complications of the reduction of cervical spine dislocations. J Bone Joint Surg (Br) 1993;75:403-409.

9. Olerud C, Jónsson H. Compression of the cervical spine cord after reduction of fracture dislocations. Report of 2 cases. Acta Orthop Scand 1991;62:599-601.

10. Rizzolo SJ, Piazza MR, Cotler JM, Balderston RA, Shaefer DFA . Intervertebral disc injury complicating cervical spine trauma. Spine (Phila Pa 1976) 1991;16(6 Suppl):S187-89.

11. Vaccaro AR, Falatyn SP, Flanders AE, et al. Magnetic resonance evaluation of the intervertebral disc, spinal ligaments, and spinal cord before and after closed traction reduction of cervical spine dislocations. Spine (Phila Pa 1976) 1999;24:1210-17.

12. Fleming M, Westgarth-Taylor T, Candy S, Dunn R. Interobserver reliability in determining the presence of hazardous disc disruption in flexion distraction injuries of the C-spine: an MRI study. SA Ortho J 2014:13(4):14-18.

13. Grant GA, Mirza SK, Chapman JR, et al. Risk of early closed reduction in cervical spine subluxation injuries. J Neurosurg 1999;90:13-18.

14. Grauer JN, Vaccaro AR, Lee JY, et al. The timing and influence of MRI on the management of patients with cervical facet dislocations remains highly variable: a survey of members of the Spine Trauma Study Group. J Spinal Disord Tech 2009;22:96-99.

15. Toit AB, Dunn R, Town C. Bifacet dislocations of cervical spine: acute management and outcome. SA Ortho J. 2008;7(4):30-36.

16. Storm M, Surgery $O$, Dunn R, et al. Management of unilateral cervical facet dislocations - a review of 49 cases. SA Ortho J 2007;6(3):14-22. 
17. Kwon BK, Beiner J, Grauer JN, Albert TJ. Anterior/posterior operative reduction of cervical spine dislocation: techniques and literature review. Curr Opin Orthop 2003;14:193-99. doi: 10.1097/00001433-200306000-00012.

18. Wiseman DB, Bellabarba C, Mirza SK, Chapman J. Anterior versus posterior surgical treatment for traumatic cervical spine dislocation. Curr Opin Orthop 2003;14:174-81. doi: 10.1097/00001433-200306000-00009.

19. Gliklich RE, Dreyer NA. Data Collection and Quality Assurance. Agency for Healthcare Research and Quality. Outcome Sciences, Inc., d/b / a Outcome Rockville (MD): Agency for Healthcare Research and Quality (US); 2010 Sep.Publication No.: 10-EHC049.

20. Grant GA, Mirza SK, Chapman JR, et al. Risk of early closed reduction in cervical spine subluxation injuries. $J$ Neurosurg Spine 1999;90:13-18. doi: 10.3171/spi.1999.90.1.0013.

21. Rizzolo SJ, Piazza MR, Cotler JM, et al. Intervertebral disc injury complicating cervical spine trauma. Spine (Phila. Pa. 1976). 1991;16:187-89.
22. Cotler JM, Herbison GJ, Nasuti JF, et al. Closed reduction of traumatic cervical spine dislocation using traction weights up to 140 pounds. Spine (Phila Pa 1976) 1993;18:386-90.

23. Benzel EC, Hart BL, Ball PA, et al. Magnetic resonance imaging for the evaluation of patients with occult cervical spine injury. J Neurosurg 1996;85:824-29. doi: 10.3171 /jns.1996.85.5.0824.

24. Wilson JR, Vaccaro A, Harrop JS, et al. The impact of facet dislocation on clinical outcomes after cervical spinal cord injury: results of a multicenter North American prospective cohort study. Spine (Phila Pa 1976) 2013;38:97-103. doi: 10.1097/BRS.0b013e31826e2b91.

This article is also available online on the SAOA website (www.saoa.org.za) and the SciELO website (www.scielo.org.za). Follow the directions on the Contents page of this journal to access it. 